\title{
Tipologías distintivas de respuestas ante el estrés académico en universitarios: un análisis de clasificación
}

\section{Distinctive types of responses to academic stress in university students: a classification analysis}

\author{
Carmen Caballero-Domínguez ${ }^{1}$ D, Yuly Suarez-Colorado² ${ }^{\text {D }}$
}

Tipología: Artículo de investigación científica y tecnológica

Para citar este artículo: Caballero-Domínguez C, Suarez-Colorado Y. Tipologías distintivas de respuestas ante el estrés académico en universitarios: un análisis de clasificación. Duazary. 2019 mayo; 16(2): 245-257. Doi: https://doi. org/10.21676/2389783X.2748

Recibido en mayo 10 de 2018

Aceptado en diciembre 21 de 2018

Publicado en línea en febrero 15 de 2019

\section{RESUMEN}

El objetivo de este estudio fue establecer las características distintivas de las respuestas ante el estrés de los estudiantes universitarios del área de la salud. Se utilizó un diseño transversal con una muestra aleatoria y estratificada de 820 estudiantes de Ciencias de la Salud de una Universidad del Caribe Colombiano. Los instrumentos utilizados fueron el MBI-SS, el UWES-S, EEC-M, el STAI y el de depresión de Bechk. Los resultados señalan que los universitarios presentan diferentes tipologías de respuesta ante el estrés, mediadas por relaciones diversas entre el burnout académico y el engagement con depresión, ansiedad, estrategias de afrontamiento y apoyo social-familiar, que configuran patrones complejos de interrelaciones mutuas entre dichas variables, conformando tres grupos distintivos de estrés: "los pilos". estudiantes con niveles alto de engagement; "los implicados y estresados"; y "los quemados", estudiantes con niveles alto de burnout.

Palabras clave: burnout académico; engagement académico; estudiantes universitarios; estudio transversal.

\section{ABSTRACT}

The objective of this study was to establish the distinctive characteristics of the responses to the stress of university students in the area of health. A cross-sectional design with a random and stratified sample of 820 students of Health Sciences of a Colombian Caribbean University was used. The instruments used were the MBI-SS, the UWES-S, EEC-M, the STAI and the depression of Bechk. The results indicate that university students present different types of response to stress, mediated by diverse relationships between academic burnout and engagement with depression, anxiety, coping strategies and social-family support, which shape complex patterns of mutual interrelations between the variables, forming three distinct groups of stress, "los pilos" students with high levels of engagement, "those involved and stressed", and "burned" students with high levels of burnout.

Keywords: Academic Burnout; Academic Engagement; University Students; Cross-Sectional Study.

1. Universidad del Magdalena. Santa Marta, Colombia. Correo: ccaballero@unimagdalena.edu.co - http://orcid.org/0000-0003-3730-2750

2. Universidad del Magdalena. Santa Marta, Colombia. Correo: ysuc91@gmail.com - http://orcid.org/0000-0001-7715-3556a 


\section{INTRODUCCIÓN}

os jóvenes ingresan generalmente a la uniLversidad con la expectativa de alcanzar un posicionamiento profesional y reconocimiento social que les garantice estabilidad laboral; sin embargo, muchos no cuentan con los recursos psicológicos, económicos y familiares que les permitan manejar adecuadamente los eventos estresores y las dificultades propias del proceso de enseñanza-aprendizaje. Estos eventos predisponen y/o agudizan respuestas de estrés; si bien los niveles bajos de estrés pueden movilizar constructivamente al individuo, los altos niveles pueden contribuir al surgimiento de respuestas emocionales, cognitivas y conductuales que pueden repercutir negativamente en la salud y la adaptación de los estudiantes al contexto académico y a su proceso formativo ${ }^{1}$. A estas manifestaciones en un nivel crónico, maligno e insidioso se les han reconocido como burnout académico ${ }^{2,3}$.

La investigación sobre el síndrome de burnout ha tomado un nuevo curso, centrado en estudiar su opuesto: el engagement. Esta perspectiva se fundamenta en la psicología positiva, que se preocupa por el buen funcionamiento y las fortalezas del individuo frente a los eventos de estrés e intenta, por esta vía, superar los enfoques preocupados por estudiar el déficit y la patología. Desde este punto de vista surge el engagement, asociado con los conceptos de "vinculación psicológica" e "implicación", y es considerado como un "estado psicológico positivo caracterizado por vigor, dedicación y absorción frente a los estudios".

El síndrome de burnout es causa de disfunción mental ${ }^{7}$, y se asocia con síntomas de depresión, burnout y consumo de licor en estudiantes de medicina ${ }^{8}$ con depresión y ansiedad ${ }^{9}$. En esta perspectiva, Caballero ${ }^{1}$ señala que a mayores niveles de burnout académico aumenta la probabilidad de mayor presencia de indicadores de afectación de la salud mental en el estudiante (mayor tensión y agotamiento emocional, ansiedad y tendencia a la depresión), lo que establece un circuito recursivo entre dichas dimensiones estructurales del síndrome y las perturbaciones emocionales mencionadas, resultando un feedback negativo y progresivamente autorreforzante entre ellas ${ }^{2}$. Por su parte, el engagement se asocia con baja depresión y ansiedad de rasgo y de estado ${ }^{9}$, con menor percepción de estrés, mayor inteligencia emocional ${ }^{10}$.

Los anteriores hallazgos ponen en evidencia que los estudiantes universitarios ante el estrés académico pueden tener una variedad de respuestas como el disconfort y vivencias de malestar emocional (burnout) o de bienestar e implicación (engagement) ante los eventos estresores, y a su vez pueden mediar la satisfacción y la salud del estudiante.

\section{Relación del burnout y el engagement con estrategias de afrontamiento}

Diversos estudios sobre el síndrome de burnout y engagement en el ámbito organizacional/laboral son consistentes sobre la importancia de las estrategias de afrontamiento en la determinación del desarrollo y evolución del síndrome en dichos ámbitos, en la medida que dichas estrategias resulten eficaces o no para manejar y resolver satisfactoriamente las demandas específicas y las emociones concomitantes a los estresores ${ }^{11}$. 
Lazarus y Launier ${ }^{12}$ consideran que el afrontamiento consiste en realizar esfuerzos cognitivos, afectivos y conductuales para manejar (dominar, tolerar, reducir o minimizar) las demandas ambientales o internas, así como los conflictos entre estas. De acuerdo con Lazarus y Folkman ${ }^{13}$ el afrontamiento se clasifica con base en las siguientes categorías: a) afrontamiento dirigido a la emoción o valoración de la situación; b) afrontamiento dirigido a la solución de problemas.

Diversos autores coinciden en señalar que las estrategias de afrontamiento centradas en la emoción o en la evitación facilitan la aparición del burnout mientras que las estrategias activas y centradas en la solución de los problemas previenen el síndrome de burnout y se asocia con el engagement ${ }^{1,14}$.

\section{Relación del síndrome de burnout y engagement con apoyo social-familiar}

El apoyo social-familiar hace referencia a la ayuda real o percibida que el estudiante obtiene de sus redes sociales significativas y, en consecuencia, se constituye en una fuente de recursos para el afrontamiento de demandas (académi- cas y no académicas). El apoyo social centrado fundamentalmente en las funciones de estímulo, comprensión, orientación y apoyo emocional que el estudiante percibe y valora de familiares, amigos y otros significativos se relaciona con su desempeño académico y las circunstancias que sus estudios le deparan 15.

En el ámbito académico se ha encontrado que el apoyo social podría actuar, además, como un potenciador en el logro de un desempeño satisfactorio de los estudiantes 15 , ya que contribuiría a que afronten los estresores propios de su ambiente con mayores probabilidades de éxito16. Igualmente, en esta dirección existen estudios sobre las relaciones entre salud mental, estrés académico, burnout académico y el apoyo social percibido en estudiantes universitarios ${ }^{17-19}$.

Este estudio tiene como objetivo caracterizar las tipologías distintivas de respuestas de los estudiantes ante el estrés académico, a partir de la relación entre el burnout y el engagement, y en el marco de esta relación analizar sus vinculaciones con depresión, ansiedad de rasgo y de estado, estrategias de afrontamiento, variables del desempeño académico, y variables del contexto social-familiar.

\section{MATERIALES Y MÉTODOS}

\section{Participantes}

Tabla 1. Frecuencias de características sociodemográficas.

\begin{tabular}{|l|l|c|c|}
\hline \multicolumn{2}{|l|}{} & Frecuencia & Porcentaje \\
\hline \multirow{2}{*}{ Género } & Masculino & 171 & 20,9 \\
\cline { 2 - 4 } & Femenino & 649 & 79,1 \\
\hline \multirow{2}{*}{$\begin{array}{l}\text { Xdad } \\
\text { Dt } \mathbf{2 4}\end{array}$} & Entre 17-20 años & 326 & 39,8 \\
\cline { 2 - 4 } & Entre 21-24 años & 435 & 53,0 \\
\cline { 2 - 4 } & Entre 25-27 años & 59 & 7,2 \\
\hline
\end{tabular}


La muestra estuvo conformada por 887 estudiantes seleccionados a través de un muestreo aleatorio y estratificado por semestre, que arrojó una muestra de 941 estudiantes, distribuidos así: 38\% (356) del programa de Medicina, 36\% (364) de Psicología, 26\% (241) de Enfermería. La pérdida muestral se representó en 67 (7,55\%) participantes, por omisión de respuesta, para un tamaño final de 820 sujetos (Tabla 1).

\section{Instrumentos}

El síndrome de burnout académico: se evaluó por el Cuestionario de Burnout Académico de Maslach Burnout Inventory-Student Survey [MBI-SS], ${ }^{6}$ adaptado a la población de estudiantes universitarios colombianos por Hederich y Caballero. ${ }^{20}$ Evalúa a través de 14 ítems las dimensiones de burnout académico: agotamiento cinismo y eficacia académica.

El engagement académico: se evaluó con la Escala de Engagement Académico [UWES-S], versión modificada del UWES, desarrollada y adaptada por Schaufeli et $a l^{6}$ para ser usado en la población de estudiantes universitarios. El UWES-S evalúa a través de 17 ítems las tres dimensiones del engagement académico: vigor, dedicación y absorción.

Los ítems de los cuestionarios MBI-SS y UWES-S puntúan en una escala de frecuencia de 7 puntos que va desde 0 (nunca) a 6 (siempre). Los índices de consistencia interna de cada una de las dimensiones de estos cuestionarios fueron satisfactorios, tal como se evidencia en la Tabla 2.

Tabla 2. Datos descriptivos, alfa de Cronbach de las variables del burnout - engagement

\begin{tabular}{|c|l|l|c|c|c|c|}
\hline \multirow{2}{*}{ Escalas } & \multicolumn{1}{|c|}{ Subescala } & $\begin{array}{c}\mathbf{N}^{\circ} \text { de } \\
\text { ítems }\end{array}$ & $\begin{array}{c}\text { Alfa de } \\
\text { Cronbach }\end{array}$ & Media & $\begin{array}{c}\text { Desv. } \\
\text { Típ. }\end{array}$ & $\begin{array}{c}\mathbf{N}^{\circ} \text { de } \\
\text { modalidades }^{*}\end{array}$ \\
\hline \multirow{4}{*}{ Burnout académico } & Agotamiento & 3 & 0,77 & 11,71 & 5,40 & 3 \\
\cline { 2 - 7 } & Cinismo & 3 & 0,718 & 2,18 & 3,19 & 3 \\
\cline { 2 - 7 } & Ineficacia académica & 3 & 0,780 & 7,93 & 4,93 & 3 \\
\hline \multirow{3}{*}{ Engagement académico } & Vigor & 3 & 0,756 & 2,63 & 0,51 & 3 \\
\cline { 2 - 7 } & Dedicación & 3 & 0,739 & 2,67 & 0,49 & 3 \\
\cline { 2 - 7 } & Absorción & 3 & 0,739 & 2,34 & 0,51 & 3 \\
\hline
\end{tabular}

${ }^{\star}$ El número de las modalidades o de los valores corresponde a los niveles o categoría (nivel alto, nivel medio y nivel bajo) de las variables activas.

Estrategias de Afrontamiento: se evaluaron mediante la Escala Estrategias de Afrontamiento - Modificada [EEC-M] adaptada en Colombia por Londoño et $a l^{21}$. Todos los ítems del cuestionario EEC-M puntúan en una escala de frecuencia de 6 puntos, que va desde 1 (nunca) a 6 (siempre). La prueba contiene 69 ítems y 12 dimensiones: búsqueda de apoyo social, espera, religión, evitación emocional, búsqueda de apoyo profesional, reacción agresiva, evitación cognitiva, reevaluación positiva, expresión de la dificultad de afrontamiento, solución de problema, negación y autonomía. Los ítems son puntuados en una escala Likert con opciones de respuesta que van de nunca (1) a siempre (6). Los índices de consistencia interna de cada una de las dimensiones fueron satisfactorios, tal como se evidencia en la Tabla 3. 
Ansiedad de rasgo y de estado: se exploró a través del Cuestionario Ansiedad Estado-Rasgo (STAI). Esta escala comprende 40 ítems, que evalúan Ansiedad de Estado y Ansiedad Rasgo, mediante una escala Likert que va de (1) no en lo absoluto y (4) mucho. Esta escala arrojó un nivel de consistencia interna satisfactorio (Tabla 3 ).
Depresión: se indagó mediante el Inventario de depresión de Beck, Rush, Shaw \& Emery, (1961). Este instrumento consta de 21 ítems que evalúan síntomas cognitivos, fisiológicos y conductuales característicos de la depresión, mediante una escala Likert que va de (0) y (3) ausencia/presencia del síntoma.

Tabla 3. Datos descriptivos, Alfa de Cronbach de las variables del sujeto.

\begin{tabular}{|l|c|c|c|c|c|}
\hline \multicolumn{1}{|c|}{ Componentes } & $\begin{array}{c}\mathbf{N}^{\circ} \text { de } \\
\text { ítems }\end{array}$ & $\begin{array}{c}\text { Alfa de } \\
\text { Cronbach }\end{array}$ & Media & $\begin{array}{c}\text { Desv. } \\
\text { Típ. }\end{array}$ & $\begin{array}{c}\mathbf{N}^{\circ} \text { de } \\
\text { modalidades }^{*}\end{array}$ \\
\hline Depresión & 21 & 0,898 & 6,85 & 7,105 & 3 \\
\hline Ansiedad rasgo & 20 & 0,898 & 39,72 & 11,393 & 3 \\
\hline Ansiedad estado & 20 & 0,914 & 38,19 & 10,940 & 3 \\
\hline Búsqueda de Apoyo Social (BAS) & 7 & 0,871 & 24,19 & 7,442 & 3 \\
\hline Espera (ESP) & 9 & 0,841 & 23,14 & 7,579 & 3 \\
\hline Religión (REL) & 7 & 0,840 & 2,19 & 0,596 & 3 \\
\hline Evitación Emocional (EE) & 8 & 0,797 & 23,02 & 6,923 & 3 \\
\hline Búsqueda de Apoyo Profesional (BAP) & 5 & 0,895 & 3,60 & 7,058 & 3 \\
\hline Reacción Agresiva (RA) & 5 & 0,726 & 12,87 & 6,066 & 3 \\
\hline Evitación Cognitiva (EC) & 5 & 0,617 & 16,03 & 4,896 & 3 \\
\hline Reevaluación Positiva (EP) & 5 & 0,801 & 18,01 & 4,930 & 3 \\
\hline Expresión de la dificultad de afrontamiento (EDA) & 4 & 0,604 & 10,41 & 3,433 & 3 \\
\hline Solución de problema & 9 & 0,844 & 34,34 & 7,999 & 3 \\
\hline Negación (NEG) & 3 & 0,543 & 8,49 & 2,743 & 3 \\
\hline Autonomía (AUT) & 2 & 0,519 & 5,21 & 2,00 & 3 \\
\hline
\end{tabular}

*El número de las modalidades o de los valores corresponde a los niveles o categoría (nivel alto, nivel medio y nivel bajo) de las variables activas.

Tabla 4. Datos descriptivos, Alfa de Cronbach de las variables del contexto, sociales y familiares.

\begin{tabular}{|c|l|c|c|c|c|c|}
\hline \multicolumn{1}{|c|}{ Escalas } & \multicolumn{1}{|c|}{ Subescala } & $\begin{array}{c}\mathbf{N}^{\circ} \text { de } \\
\text { ítems }\end{array}$ & $\begin{array}{c}\text { Alfa de } \\
\text { Cronbach }\end{array}$ & Media & $\begin{array}{c}\text { Desv. } \\
\text { Típ. }\end{array}$ & $\begin{array}{c}{ }^{\star} \mathbf{N}^{\circ} \text { de } \\
\text { modalidades }\end{array}$ \\
\hline \multirow{2}{*}{ Contexto social familiar } & Apoyo social (AS) & 4 & 0,785 & 7,02 & 3,41 & 3 \\
\cline { 2 - 7 } & $\begin{array}{l}\text { Relaciones con la familia } \\
\text { y amistades (RF) }\end{array}$ & 3 & 0,642 & 11,25 & 3,00 & 3 \\
\hline
\end{tabular}

${ }^{\star}$ El número de las modalidades o de los valores corresponde a los niveles o categoría (nivel alto, nivel medio y nivel bajo) de las variables activas. 
Apoyo social y relaciones con la familia y amistades: se evaluó mediante una escala construida por los investigadores del presente estudio, que evalúa la percepción de los estudiantes sobre el contexto interpersonal como el familiar y de amistad. Todos los ítems del contexto socio-familiar puntúan en una escala de frecuencia de 4 puntos que va desde 0 (nunca) a 3 (siempre). Los índices de consistencia interna de cada una de las dimensiones de estos cuestionarios se consideran adecuados, tal como se evidencia en la Tabla 4.

\section{Procedimiento}

Los instrumentos fueron diligenciados por los sujetos de manera individual, una vez que se les proporcionó información acerca del estudio y firmado el consentimiento informado. El procesamiento y análisis estadístico de los datos se realizó mediante el software SPAD versión 4,5 .

Previo al Análisis de Clasificación (AC) de los individuos, fue necesario explorar el entramado asociativo posible del burnout y engagement mediante Análisis de Correspondencias Múltiples (ACM). En síntesis, el ACM permite una visión global de las variables en un reducido número de ejes factoriales que facilitan interpretar de manera rápida y sugerente las relaciones de interdependencia existentes entre las variables activas e ilustrativas ${ }^{22}$. Las variables del síndrome de burnout y engagement, y en particular sus modalidades, se tomaron como variables activas; estas son las que intervienen en la definición de los factores o dimensiones. Desde esta perspectiva, los factores finalmente son variables no observadas que se construyen como combinación lineal de las modalidades de las variables seleccionadas como variables activas.

Tabla 5. Histograma de los autovalores de los primeros 12 factores del estudio.

\begin{tabular}{|l|l|l|l|l|}
\hline No & $\begin{array}{c}\text { Valor } \\
\text { Propio }\end{array}$ & \% Varianza & \% Acumulado & \\
\hline 1 & 0,4373 & 21,87 & 21,87 & $* * * * * * * * * * * * * * * * * * * * * * * * * * * * * * * * * * * * * * * * * * * * * * * * * *$ \\
\hline 2 & 0,2498 & 12,49 & 34,36 & $* * * * * * * * * * * * * * * * * * *$ \\
\hline 3 & 0,2075 & 10,38 & 44,73 & $* * * * * * * * * * * *$ \\
\hline 4 & 0,2015 & 10,07 & 54,80 & $* * * * * * * * * *$ \\
\hline 5 & 0,1690 & 8,45 & 63,26 & $* * * * * * * * *$ \\
\hline 6 & 0,1490 & 7,45 & 70,70 & $* * * * * * *$ \\
\hline 7 & 0,1390 & 6,54 & 77,25 & $* * * * * *$ \\
\hline 8 & 0,1209 & 6,04 & 83,29 & $* * * * *$ \\
\hline 9 & 0,1147 & 5,73 & 89,03 & $* * * *$ \\
\hline 10 & 0,0913 & 4,56 & 93,59 & $* * * *$ \\
\hline 11 & 0,0708 & 3,54 & 97,13 & $* * *$ \\
\hline 12 & 0,0574 & 2,87 & 100,0 & $* * *$ \\
\hline
\end{tabular}


En el análisis del histograma, tal y como se observa en la Tabla 5, se encontraron 12 factores; se seleccionó el primer factor que se destaca por tener un valor propio de 0,4373 , que explica el $21,87 \%$ de la inercia, que se polariza en la trayectoria de las diferentes modalidades del burnout y del engagement académicos. Las modalidades que contribuyen de forma especialmente alta, de acuerdo con su contribución acumulada, son: en mayor grado, dedicación, con $23,5 \%$; luego, ineficacia académica, con 23,1\%; vigor, con $20,8 \%$, y cinismo, con $19,5 \%$; en menor grado se encuentra absorción, con $8,7 \%$, y agotamiento, con $4,3 \%$. La posición de las modalidades del burnout y del engagement en el plano factorial se le denominó en el presente estudio "Respuesta de los estudiantes ante el estrés".

Por su parte, para caracterizar el plano factorial "Respuesta de los estudiantes ante el estrés académico", se agruparon las variables ilustrativas, y en particular sus modalidades, de acuerdo a información sobre su desempeño académico (ejemplo: materias perdidas, semestres perdidos, promedio académico) e indicadores compromiso de salud mental (ejemplo: depresión y ansiedad), estrategias de afrontamiento (ejemplo: solución de problema, evitación cognitiva, espera, evitación emocional y cognitiva, religión...) y variables del contexto organizacional universitario y social (conflicto y ambigüedad del rol, dinámica negativa del trabajo académico, apoyo social y familiar...).

Una vez realizado el ACM, se llevó a cabo el AC que permitió establecer las tipologías distintivas de las respuesta de los estudiantes, basada en las coordenadas factoriales del plano factorial del continuo burnout-engagement o "respuestas de los estudiantes ante el estrés académico", lo que permitió caracterizar las tipologías de acuerdo con los indicadores de compromiso de la salud mental, de las estrategias de afrontamiento, del desempeño académico, variables de los contextos organizacional universitario y social-familiar que caracterizan mejor a cada grupo ${ }^{22}$.

Se tomó la caracterización del segundo nivel de clasificación que define tres grandes grupos, que caracteriza dos grupos claramente diferenciados - marcados por las dos grandes ramas que dividen el grupo total- que representan al grupo de estudiantes con burnout y el grupo de estudiantes con engagement, que se ubican a lo largo del continuo polar de las "respuestas de los estudiantes ante el estrés" y un tercer grupo que caracteriza los estudiantes con valores intermedios que representan a la gran mayoría de la población. La definición de los tres grupos de respuestas de los estudiantes ante el estrés polariza y combina los dos ejes factoriales en todas las posibilidades, los cuales se tendrán en cuenta para el análisis de las tipologías de los estudiantes ante el estrés académico.

\section{Declaración sobre aspectos éticos}

Se respetó la Declaración de Helsinki e igualmente fue considerada la Resolución 8430 de 1993 del Ministerio de Salud de Colombia.

\section{RESULTADOS}

Las respuestas de estrés de los estudiantes se pueden caracterizar a partir de tres grupos identificados; dos se sitúan a lo largo de la trayectoria del factor del burnout y engagement académico, es decir, estos dos grupos guardan mayor correspondencia con las denominaciones del burnout académico (el grupo 1) y con el engagement académico (el grupo 3). El grupo 2 está caracterizado por valores medios de las diferentes modalidades de las variables ilustrativas incluidas en este estudio. A continuación, se describirá y analizará cada uno de los grupos (Figura 1). 
Figura 1. Ubicación de los tres grupos sobre el plano factorial respuestas de estrés de los estudiantes.

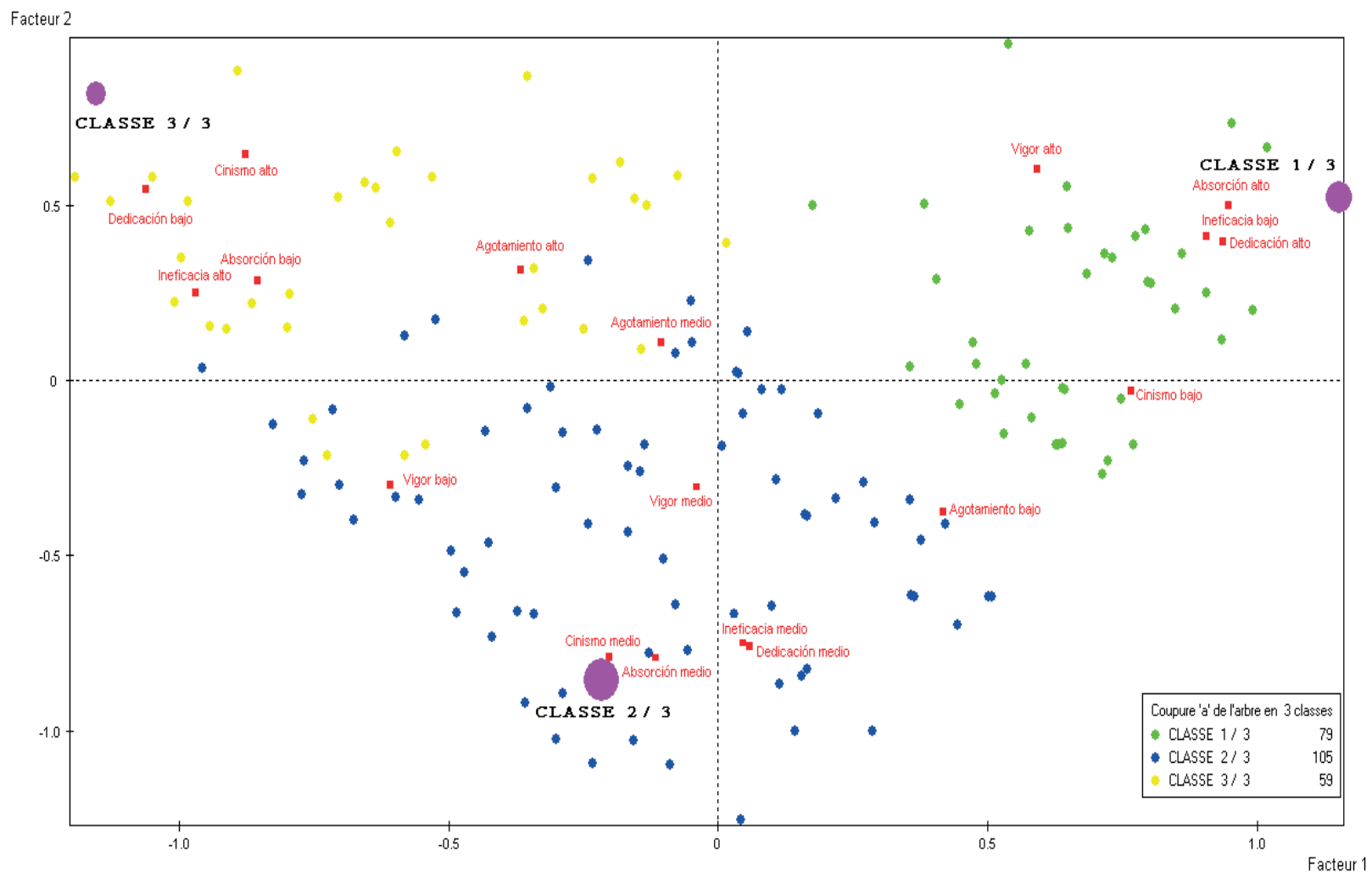

Grupo uno: Los estudiantes "pilos". Este grupo se encuentra conformado por el $32,51 \%$ de los estudiantes; en lenguaje coloquial son definidos como "pilos", y se caracterizan por presentar altos niveles de engagement $y$, por ende, presentan altos niveles de dedicación (v.test $=11.45, p<0,000$ ), alta percepción de competencia para afrontar las dificultades académicas (v.test $=10,43, p<0,000$ ), bajo cinismo (v.test $=8,28, p<0,000$ ), alta adsorción (v.test $=6,38, p<0,000)$, y por último, bajo agotamiento (v.test $=2,61, p<0,000$ ).

En cuanto al uso de las estrategias de afrontamiento que resultaron distintivas en este grupo, se puede mencionar alta solución de problema (v.test $=6,05, p<0,000$ ); alto apoyo social (v.test $=4,33, p<0,000$ ); baja religión (v.test $=3,68$, $p<0,000)$. Además, alta reevaluación positiva (v.test $=3,13, p<0,000)$ y baja reacción agresiva: (v.test $=2,34, p<0,010)$. Al analizar los indicadores de salud mental, este grupo se distingue por baja ansiedad estado (v.test $=4,96 p<0,010$ ), baja ansiedad rasgo (v.test $=3,26, p<0,001$ ), y baja depresión (v.test $=3,03, p<0,010$ ). En relación con las características particulares del contexto sociofamiliar que distinguen a los estudiantes "pilos" se caracterizan por alto apoyo familiar (R.F.2) (v.test $=3,34, p<0,000)$ y social (v.test $=3,79, p<0,000)$.

Grupo dos: los estudiantes "implicados y estresados". Recurriendo al lenguaje coloquial, este grupo se distingue como estudiantes "implicados y estresados", y está conformado por 105 estudiantes, lo cual corresponde al $43,2 \%$ de la muestra. Este grupo se caracteriza por la ten- 
dencia a mantener cierta actitud negativa y de distanciamiento frente a las actividades académicas (v.test $=7,89, p<0,000$ ). Se encuentran levemente implicados, comprometidos y tienen energía para llevar a cabo las actividades académicas (v.test=6,82, $p<0,000$ ). Presentan ciertas dudas acerca de su competencia para superar los obstáculos y dificultades (v.test=5,37, $p<0,000$ ). Además, no logran estudiar durante un largo periodo de tiempo con una sensación de felicidad y de disfrute (v.test $=4,22, p<0,000$ ).

En cuanto al uso de estrategias de afrontamiento, se encontró que las modalidades que predominaron fueron la tendencia a usar, en nivel medio, el rezo y la oración (v.test $=3,63, p<0,000$ ), asimismo, no piensan en el problema y se alejan de la situación de manera temporal (v.test $=3,20$, $p<0,000)$ como medidas para tolerar o soportar el estado emocional, y no buscan ayuda en otras personas para el manejo de las emociones y para solucionar los problemas (v.test $=2,38, p<0,000$ ).

Por otra parte, entre las variables académicas, la modalidad que caracterizó al grupo de estudiantes "implicados y estresados" se halló el hecho de cursar séptimo semestre (v.test $=2,70, p<0,003$ ). En relación con las variables del contexto social familiar, se destaca, a diferencia de los estudiantes "pilos", que no tienen conflictos con la familia (v.test $=2,45, p<0007$ ) y consideran que en general las relaciones entre los compañeros de clase son adecuadas (v.test $=2,33, p<0,010)$.

Grupo tres: los "estudiantes quemados". Este grupo estuvo conformado por 59 estudiantes $(24,28 \%)$ y es una antítesis de los grupos anteriores, especialmente del grupo "implicado". En él se ubican exclusivamente los estudiantes que presentan niveles medios y altos de burnout, por lo tanto, manifiestan una actitud negativa de desinterés y de distanciamiento respecto a las actividades académicas (v.test $=13,21, p<0,000$ ). Por consiguiente, muestran escasa dedicación y compromiso (v.test $=9,86, p<0,000$ ) y una percepción elevada de ineficacia ante los estudios (v.test $=7,26, p<0,000$ ). A su vez, presentan niveles altos de depresión (v.test $=5,59, p<0,000)$ y agotamiento físico y/o emocional (v.test $=4,83$, $p<0,000$ ), de ansiedad estado (v.test $=4,64$, $p<0,000$ ), rasgo (v.test $=4,19, p<0,000)$ y escasa energía para realizar las actividades académicas (test $=4,08, p<0,000)$.

En relación con las estrategias de afrontamiento, los estudiantes "quemados" bajo uso de la solución del problema (v.test $=3,36, p<0,000$ ), alto uso de reacción agresiva (v.test $=3,23, p<0,000$ ), bajo uso reevaluación positiva (v.test $=3,15$, $p<0,000)$ y alto uso de expresión dificultad de afrontamiento (v.test $=2,39, p<0,000$ ).

En cuanto a las características particulares del contexto social-familiar distingue a los estudiantes "quemados" de los anteriores grupos que tienen relaciones conflictivas con la familia (v.test=2,92, $p<0,002$ ), no cuentan con la aprobación y estímulo por parte de esta para la realización de la carrera escogida (v.test $=27,1$, $p<0,003)$ y se muestran indiferentes al apoyo emocional brindado de la familia ante las dificultades académicas (v.test $=3,18, p<0,001$ ).

\section{DISCUSIÓN}

El análisis de clasificación ha mostrado tres grupos que polarizan el continuo polar de las "respuestas de los estudiantes ante el estrés". Dos grupos muestran características opuestas, no solamente ante el estrés, sino también en los indicadores de compromiso de la salud mental, en el afrontamiento y en la percepción ante las variables del contexto social y familiar. 
No obstante, el último grupo se caracteriza por presentar niveles medios en las variables anteriormente mencionadas.

Desde esta perspectiva, vemos que los estudiantes "pilos" son comprometidos, dedicados e implicados, no presentan indicadores de afectación de la salud mental, tienden a utilizar estrategias de afrontamiento centradas en la solución, en la reevaluación positiva y en la búsqueda de apoyo social. Además, no utilizan la agresión como medio para solucionar los problemas. En lo que respecta a las variables académicas, el promedio académico es bueno, lo cual diferencia a este grupo de los otros dos. En cuanto a las variables del contexto social familiar cuentan con redes de apoyo social estables con los familiares y compañeros.

Por su parte, los "quemados" se encuentran exhaustos, agotados, con una actitud de distanciamiento y autosabotaje, poseen creencias de ineficaces ante las actividades académicas, acompañadas de niveles bajos de compromiso e implicación ante los estudios, además de niveles altos de ansiedad rasgo y estado. En relación con el afrontamiento del malestar emocional asociado al estrés, no recurren a buscar ayuda, ni profesional ni social, no expresan las emociones para disminuirla y solucionar los problemas. Otra variable que caracteriza a este grupo es la percepción de no contar con oportunidades laborales que le permitan alcanzar expectativas de desarrollo profesional y social. Al analizar en este grupo las variables del contexto social mayor, se destacaron las dificultades en las relaciones con la familia y los amigos e inadecuado apoyo social.

Los "implicados y estresados" se caracterizan por estar relativamente implicados, comprometidos, cínicos y poco vigorosos, respuestas que pueden acercarse a manifestaciones propias del estrés, sin que esto necesariamente signifique que presenten el síndrome de burnout. Por otra parte, muchos de estos estudiantes se encuentran en séptimo semestre, en el que se da inicio a las prácticas. En relación con los factores del contexto social familiar, este grupo guarda cierta similitud con el grupo de los estudiantes implicados en la medida que tienden a percibir de manera negativa las variables las relaciones con los amigos y familia. En cuanto a las estrategias de afrontamiento, se observa de manera particular la tendencia a no pensar sobre las situaciones que les generan incomodidad y no buscar ayuda social para solucionar los problemas.

A manera de síntesis, y al analizar los elementos en común de los tres grupos, se destacan el uso de las estrategias de afrontamiento de religión y de apoyo social y, de manera particular, lo que los diferencia es la tendencia de los estudiantes implicados en utilizar las estrategias de solución de problemas y reevaluación positiva; en los implicados y estresados es la evitación cognitiva, y en los estudiantes quemados es el uso de la estrategia de reacción agresiva. Esto muestra cómo estos estudiantes utilizan diversas estrategias para manejar las demandas particulares y las emociones asociadas a eventos vitales, ante los que pueden experimentar altos niveles de estrés y comprometer su bienestar psicológico, su salud mental, satisfacción con su rol y desempeño académico, que, por lo demás, varían en función de los recursos psicológicos que amortiguan o predisponen el síndrome de burnout.

En cuanto al papel de la depresión y ansiedad en las respuestas ante el estrés académico, los hallazgos permiten evidenciar que el burnout académico (grupo "los quemados") tiende a acompañarse de síntomas que comprometen la salud mental de los estudiantes universitarios, como ansiedad y, en menor medida, depresión, lo que nos lleva a resaltar y confirmar su comorbilidad 
con ansiedad; hallazgos que adquieren mayor notoriedad debido a que el engagement (grupo "los pilos") no se asoció con ninguno de estos indicadores de perturbación de salud mental. Por lo tanto, podemos considerar al burnout académico como un buen predictor de síntomas de ansiedad en los estudiantes, $y$, factiblemente, un factor de riesgo para el desarrollo de condiciones de mayor compromiso de salud mental por ansiedad clínicamente significativa. Lo anterior permite confirmar los planteamientos de Reyes et $a l^{8}$, Caballero et $a l^{9}$ y Arango et a ${ }^{23}$. Además, estos resultados señalan el carácter difuso, insidioso y evolutivo del síndrome, que puede cursar con y hacia formas manifiestas de ansiedad en contraste con las respuestas ante el estrés caracterizado por el engagment, que se asoció con menor afectación a nivel de la salud mental ${ }^{9}$.

Por otra parte, es necesario señalar la relación del contexto social-familiar que configura patrones complejos de interrelación e implica interrelaciones mutuas y complejas con los síndromes de burnout (grupo de "los quemados"), de engagement (grupo "los implicados") y del estrés (grupo de "los implicados y estresados"). Desde esta perspectiva, vemos que dichos factores no afectan de igual manera la salud y el desempeño de los estudiantes y/o de los grupos; lo cual puede estar mediado por la percepción de amenaza de la capacidad del individuo para controlar y afrontar el estrés, del apoyo social y de las relaciones con la familia y amigos ${ }^{24}$.

Al analizar la relación de las estrategias de afrontamiento con las respuestas del estrés académico, los resultados destacan las estrategias emotivas centradas en la emoción en el grupo de estudiantes "quemados", en tanto que las estrategias cognitivas y activas centradas en la resolución del problema prevalecieron en el grupo de los "pilos". En consecuencia, el estilo de afrontamiento media las respuestas del estrés, tal como señalan Lazarus y Folkman ${ }^{13}$, actuando como variable protectora o, en su defecto, de riesgo ante las exigencias y dificultades propias del proceso de aprendizaje a las que se enfrentan los estudiantes "quemados", "implicados" y los "implicados y estresados". Estos planteamientos están en la dirección de los hallazgos de diversas investigaciones sobre el afrontamiento del estrés en estudiantes universitarios, que señalan que los estudiantes utilizan diversas estrategias para manejar las demandas particulares y las emociones asociadas a eventos vitales estresantes, académicos y no académicos, que podrían comprometer su bienestar psicológico, su salud mental, la satisfacción con su rol y el desempeño académico $^{1,14}$.

\section{CONCLUSIÓN}

En síntesis, en el análisis de las respuestas ante el estrés del estudiante universitario se establecen diferentes modalidades de respuestas mediadas por relaciones de diversas variables, como depresión, ansiedad, estrategias de afrontamiento y apoyo social-familiar, que configuran patrones complejos que implican interrelaciones mutuas entre las mismas, lo que establece respuestas de estrés, tanto negativas y disfuncionales (burnout) como de compromiso, de disfrute $\mathrm{y}$ de felicidad (engagement). Desde otra mirada, vemos que dichas variables no afectan de igual manera la salud, el bienestar y el desempeño de los estudiantes; lo cual puede explicarse por las cogniciones (ineficacia y percepción de amenaza y vulnerabilidad) que median un determinado número de afrontamientos que va más allá de intentos específicos o situacionales para resolver, amortiguar o canalizar el estrés, lo cual se convierte en un estilo asociado a elementos característicos de la personalidad de los estudiantes. 


\section{DECLARACIÓN SOBRE CONFLICTOS DE INTERESES}

Los autores manifiestan no tener conflictos de interés.

\section{REFERENCIAS BIBLIOGRÁFICAS}

1. Caballero C. El burnout académico: Prevalencia y factores asociados en estudiantes universitarios del área de la salud de la ciudad de Barranquilla. [Disertación doctoral]. Barranquilla:Universidad del Norte; 2012.

2. Caballero C, Bresó E, González O. Burnout en estudiantes universitarios. Psicología desde el Caribe. 2015; 32(3): 425-41. DOI: http://dx.doi. org/10.14482/psdc.32.2.5742

3. Salanova M, Martínez I, Bresó E, Llorens S, Grau R. Bienestar psicológico en estudiantes universitarios: facilitadores y obstaculizadores del desempeño académico. Anales de Psicología. 2005; 21(1): 170-80. Disponible en: https://www. redalyc.org/pdf/167/16721116.pdf

4. SalanovaM,Llorens S. Estadoactualy retosfuturos en el estudio del burnout. Papeles del Psicólogo. 2008; 29(1): 59-67. Disponible en: https://www. redalyc.org/articulo.oa?id $=77829108$

5. González-Roma V, Schaufeli W, Bakker A, Lloret S. Burnout and work engagement: Independent factors or opposite poles. Journal of Vocational Behavior. 2006; 68 (1): 165-74.

6. Schaufeli WB, Martínez I, Marques A, Salanova $M$, Bakker A. Burnout and engagement in university students: A cross-national study. Journal of Cross - Cultural Psychology. 2002; 33 (5): 464-81.

7. Maslach C, Schaufeli W, Leiter M. Job Burnout. Annual Review of Psychology. 2001; 52: 397-422.
8. Reyes A, Medina M, Mesa X, Paredes Y, Barahona Y, Sierra M. Estudio de síndrome de "burnout", depresión y factores asociados en los practicantes internos del hospital escuela. Revista Facultad Ciencias Medica. 2012; 9(1): 14-20.

9. Caballero C, González O, Palacio J. Relación del burnout y el engagement con depresión, ansiedad y rendimiento académico en estudiantes universitarios. Revista Salud Uninorte. 2015; 31(1): 59-69. DOI: http://dx.doi.org/10.14482/ sun.31.1.5085

10. Durán A, Extremera N, Rey L. La Contribución de los recursos personales (Inteligencia Emocional, Core Self-Evaluation y Afectividad Positiva) para el engagement: Un análisis en estudiantes universitarios y trabajadores españoles. Ciencia y Trabajo. 2011; 42(13): 200-7.

11. Gil-Monte PR. El síndrome de quemarse por el trabajo (síndrome de burnout), aproximaciones teóricas para su explicación y recomendación para la intervención. Revista psicologíacientífica. com [Revista en la Internet]. 2000; 3(5).

12. Lazarus RS, Launier R. Stress-related transactions between person and environment. In LA Pervin, M Lewis (Eds). Perspectives in Interactional Psychology. Nueva York: Plenum Press; 1978.

13. Lazarus R, Folkman S. Estrés y procesos cognitivos. Barcelona: Martínez Roca; 1986.

14. Montoya P, Moreno S. Relación entre el síndrome de burnout, estrategias de afrontamiento y engagement. Psicología desde el Caribe. 2012; 29(1): 204-27.

15. Román C, Hernández R. Variables psicosociales y su relación con el desempeño académico de estudiantes de primer año de la Escuela Latinoamericana de Medicina. Revista Iberoamericana de Educación. 2005; 37(2): 1-8.

16. Martín I. Estrés académico en estudiantes universitarios. Apuntes de Psicología. 2007; 25 (1): 87-99. 
17. Cabello L, Koch SDA. Síndrome de desgaste profesional (Burnout) y apoyo familiar en médicos residentes de la Universidad de Los Andes. MedULA. 2001; 10(1-4): 40-7.

18. Feldman L, Goncalvez L, Chacón-Puignau G, Zaragoza J, Bagés N, De Pablo J. Relaciones entre el estrés académico, apoyo social, salud mental y rendimiento académico en estudiantes universitarios venezolanos. Univ. Psychol. 2008; 7(3): 739-51.

19. Figueras AD, Celis RR, Arroyo LM, Rayo IG. Niveles de ansiedad, depresión y percepción de apoyo social en estudiantes de Odontología de la Universidad de Chile. Revista de Psicología. 2011;20(2): 147-72.

20. Hederich C, Caballero C. Validación del cuestionario Maslach Burnout InventoryStudent Survey (MBI-SS) en contexto académico colombiano. Revista CES Psicología. 2016; 9(1): 1-15. DOI: http://dx.doi.org/10.21615/cesp.9.1.1
21. Londoño N, Henao G, Puerta I, Posada S, Arango D, Aguirre-Acevedo D. Propiedades psicometrías y validación de la Escala de Estrategias de Coping Modificada (EEC-M) en una muestra colombiana. Universitas Psychologica. 2006; 5(2): 327-49.

22. Hederich C. Estilo cognitivo en la dimensión de dependencia-independencia de campo. Influencias culturales e implicaciones para la educación. Bogotá: Universidad Pedagógica Nacional; 2007.

23. Arango S, Castaño J, Henao C, Jiménez D, López A, Páez M. Síndrome de burnout y factores asociados en estudiantes de I a X semestre de la Facultad de Medicina de la universidad de Manizales (Colombia). Archivo de Medicina. 2010; 10(2): 110-26.

24. Camacho-Ávila A, Juárez-García A, Arias Galicia F. Síndrome de burnout y factores asociados en médicos estudiantes. Cienc. Trab. 2010; 12(35): 251-4. 